OPEN ACCESS

Edited by:

Xia Xiong,

Institute of Subtropical Agriculture

(CAS), China

Reviewed by:

Bi ETan,

Hunan Agricultural University, China

Xihong Zhou,

Institute of Subtropical Agriculture

(CAS), China

*Correspondence:

Shengyi Wang

wangshengyi@caas.cn

Sufang Han

sfhan@scau.edu.cn

Specialty section: This article was submitted to Nutritional Immunology, a section of the journal

Frontiers in Immunology

Received: 22 August 2021 Accepted: 20 September 2021 Published: 05 October 2021

Citation:

Gan Z, Zhang M, Xie D, Wu X, Hong C, Fu J, Fan L, Wang S and Han S (2021)

Glycinergic Signaling in Macrophages and Its Application in MacrophageAssociated Diseases.

Front. Immunol. 12:762564. doi: 10.3389/fimmu.2021.762564

\section{Glycinergic Signaling in Macrophages and Its Application in Macrophage-Associated Diseases}

\author{
Zhending Gan ${ }^{1}$, Meiyu Zhang ${ }^{2}$, Donghui Xie ${ }^{3}$, Xiaoyan $W^{1,4}$, Changming Hong ${ }^{1}$, \\ Jian Fu ${ }^{1}$, Lijuan Fan ${ }^{1}$, Shengyi Wang ${ }^{4 *}$ and Sufang Han ${ }^{1 *}$ \\ ${ }^{1}$ College of Animal Science, South China Agricultural University, Guangzhou, China, ${ }^{2}$ College of Animal Science and \\ Technology, Guangdong Polytechnic of Science and Trade, Guangzhou, China, ${ }^{3}$ Nanchang Academy of Agricultural Sciences, \\ Nanchang, China, ${ }^{4}$ Key Laboratory of Veterinary Pharmaceutical Development, Ministry of Agricultural and Rural Affairs, \\ Lanzhou Institute of Husbandry and Pharmaceutical Science, Chinese Academy of Agricultural Science, Lanzhou, China
}

Accumulating evidences support that amino acids direct the fate decision of immune cells. Glycine is a simple structural amino acid acting as an inhibitory neurotransmitter. Besides, glycine receptors as well as glycine transporters are found in macrophages, indicating that glycine alters the functions of macrophages besides as an inhibitory neurotransmitter. Mechanistically, glycine shapes macrophage polarization via cellular signaling pathways (e.g., NF-кB, NRF2, and Akt) and microRNAs. Moreover, glycine has beneficial effects in preventing and/or treating macrophage-associated diseases such as colitis, NAFLD and ischemia-reperfusion injury. Collectively, this review highlights the conceivable role of glycinergic signaling for macrophage polarization and indicates the potential application of glycine supplementation as an adjuvant therapy in macrophage-associated diseases.

Keywords: glycine, macrophage, NF- $\mathrm{kB}$, miRNA, inflammation

\section{INTRODUCTION}

Macrophages are found in almost all tissues such as Kupffer cells in hepatocyte (1) and microglia in central nervous system (2). These macrophages engulf cellular debris, microbes, death cells and foreign substances by stretching filopodia $(3,4)$. Although the polarizations of macrophages are multiple, they are roughly polarized to two distinct subsets: classically activated (M1) phenotype and alternatively activated (M2) phenotype $(5,6)$. Macrophages polarize into M1 phenotype to perform their pathogen-scavenging function when exposed to T-helper 1 (Th 1) type cytokines or inflammatory mediators, such as interferon gamma (IFN- $\gamma$ ) and lipopolysaccharide (LPS) (7), or M2 phenotype to perform their anti-inflammatory effects, including wound healing and anti-tumor ability under conditions of exposure to Th 2 cytokines like IL-4 and IL-10 (8). Indeed, various contributors are related to the fate of macrophages. Notably, metabolism pathways and metabolites are the best examples for directing macrophage growth and survival by providing energy and substrates, and instructing functions of macrophages $(9,10)$. For example, altered amino acid metabolism [e.g., arginine metabolism (11)] is a well-accepted character to define macrophage polarization. 
Traditionally, amino acids are simply divided into two categories: essential amino acids and non-essential amino acids (12). However, many traditionally considered non-essential amino acids are not only used as substrates for protein and peptide synthesis, but also involved in regulating metabolism, signal transduction and immune responses (13). Glycine consists of one carbon $(\mathrm{C})$ atom, two hydrogen $(\mathrm{H})$ atom, one carboxylgroup $(\mathrm{COOH})$ and one amino-group $\left(\mathrm{NH}_{2}\right)$ (14). Of note, recent studies have shown that glycine affects functions of macrophage $(15,16)$. In this review, we will summarize glycinergic system in macrophages, discuss how glycine contributes to the polarization of macrophages, and list some examples that glycine mediates macrophage-associated diseases.

\section{GLYCINERGIC SYSTEM IN MACROPHAGES}

\section{Glycine Receptors in Macrophages}

Glycine is an inhibitory neurotransmitter (17), which exerts inhibitory effect by binding to glycine receptors (GlyRs) (1820). GlyRs consist of $\alpha$ subunits ( $48 \mathrm{kDa}), \beta$ subunits $(58 \mathrm{kDa})$ and a $93 \mathrm{kDa}$ subunit anchoring protein gephyrin (21). GlyRs also present in non-neuron cell membrane, such as macrophages $(20,22)$. For example, the subunits of GlyRs are found in rat Kupffer cells, splenic macrophages and alveolar macrophages, and the sequences of the cloned fragment for the GlyRs $\beta$ subunit in macrophages are more than 95\% homologous with the GlyRs from the spinal cord (22). It should be noted that the GlyRs subunits differ in various types of macrophages. For example, Kupffer cells have $\alpha 1$-subunit, $\alpha 4$-subunit and $\beta$-subunit, while $\alpha 2$-subunit, $\alpha 4$-subunit and $\beta$-subunit are found in splenic and alveolar macrophages, as well as only $\alpha 1$ subunit in the peritoneal macrophages in rats $(22,23)$. The reasons for these differences might result from the origins of macrophages (24) (embryonic origin and monocyte derivation), species of animals and even culture condition of isolated macrophages. It is also intriguing to know whether such difference presents in mouse or human macrophages. Although GlyRs have been identified on macrophages, no studies have investigated the effects of GlyRs subunits in macrophage fate decision. Notably, blocking the receptor with strychnine $(25,26)$ alleviates glycine-induced intracellular $\mathrm{Ca}^{2+}$ decrease in LPS-stimulated macrophages
$(25,27,28)$, suggesting the receptor highly shapes the fate decision of macrophages. To fully illustrate the function of GlyRs in macrophages, the comparative analysis towards expression and location of GlyRs in macrophages from different tissues and subsets (e.g., resting macrophages vs. M1 phenotype or M2 phenotype) should be performed. Then the function of GlyRs subunits in macrophage fate decision can be explored with chemical ablation or genetic manipulation.

\section{Glycine Transporters in Macrophages}

In the central nervous system (CNS), glycine is transported into cells by neutral-amino-acid transporters (NAATs, Table 1) (29, 30); however, the presence of NAATs in macrophages remains to fully explore. Interestingly, rat M1 macrophages are sensitive to NAATs substrate 2-aminoisobutyric acid (AIB) (23) and the application of methylamino-AIB inhibits glycine-induced inward currents in microglia (31), suggesting that NAATs might be expressed in macrophages. As expected, it has been demonstrated that rat peritoneal macrophages express at least one of NAATs, especially glycine transporter-1 (GlyT1) (23). Further investigations are needed to examine the expression of NAATs in mice and human macrophages.

\section{GLYCINE METABOLISM IN MACROPHAGES}

In mammals, glycine can be synthesized from serine, choline, threonine and hydroxyproline by different metabolic pathways (32). Since serine and glycine are biosynthetically linked (33), serine and its precursors can generate glycine. The conversion of serine to glycine catalyzed by serine hydroxymethyltransferase (SHMT) is the main way for glycine synthesis $(34,35)$. When glycine deficiency occurs, such as intrinsic glycine uptake capacity limitation or environmental glycine deprivation, SHMT can support glycine synthesis (36).

In addition to participating in protein synthesis, glycine is a precursor of peptides, nucleic acids as well as methyl donors. Upon LPS stimulation, the levels of intracellular glycine and glycine metabolites such as glutathione (GSH) and Sadenosylmethionine (SAM) increased (37-39). Interestingly, adding glycine to the serine-deprived medium failed to rescue IL-1 $\beta$ secretion in macrophages upon LPS stimulation (38).

TABLE 1 | Neutral-amino-acid transporters which transport glycine.

\begin{tabular}{|c|c|c|}
\hline System & Gene & Transporters (Full name and abbreviation) \\
\hline \multicolumn{3}{|c|}{ Sodium dependent NAATs } \\
\hline \multirow[t]{3}{*}{ A } & SLC38A1 & Serine acetyltransferase 1 (SAT1) \\
\hline & SLC38A2 & SAT2 \\
\hline & SLC38A4 & SAT3 \\
\hline \multirow[t]{2}{*}{ Gly } & SLC6A9 & Glycine transporter 1 (Gly 11$)$ \\
\hline & SLC6A5 & GlyT2 \\
\hline \multicolumn{3}{|c|}{ Sodium independent NAATs } \\
\hline asc & SLC7A10 & Asc Type Amino Acid Transporter 1/2 (ASC1/2) \\
\hline \multirow[t]{2}{*}{ imino } & SLC36A1 & Proton-coupled amino acid transporter 1 (PAT1) \\
\hline & SLC36A2 & Proton-coupled amino acid transporter 2 (PAT2) \\
\hline
\end{tabular}


Besides this, lack of glycine cannot affect the polarization of macrophages (39). Thus, extracellular glycine may not influence macrophage metabolism. U-[13C]-labeling shows that glycine is mainly converted from glucose and serine, and it can be subsequently converted to ADP, ATP, GSH and SAM (38). Strikingly, U-[13C]-glycine revealed a remarkable attenuation of extracellular glycine-derived GSH compared to serine (synthesis from glycine)-derived GSH (38). Moreover, supplementary glycine in serine deprived medium failed to rescue intracellular GSH in macrophage. These phenomena indicate that glycine utilization in macrophages is mainly through intracellular conversion of serine, not via exogenous glycine supply.

\section{GLYCINE REGULATES SIGNALING PATHWAYS IN MACROPHAGES}

The functions of macrophages are highly responsive to their micro-environmental stimuli. Upon the activation of Toll-like receptor (TLR) or interferon signaling, M1 macrophages arise in inflammatory to eliminate pathogens (40-42). Whereas M2 macrophages, usually found in Th2-dominated responses, can mediate helminth immunity, asthma, and allergy (43).

Among various signaling pathways regulating macrophage inflammation, $\mathrm{NF}-\kappa \mathrm{B}$ is a main contributor to orchestrate macrophage polarization (44). Glycine can prevent the activation of nuclear factor $-\kappa \mathrm{B}(\mathrm{NF}-\kappa \mathrm{B})$ by inhibiting the degradation of inhibitor of NF- $\kappa \mathrm{B}$ (I $\kappa \mathrm{B}$ ) in pro-inflammatory macrophages (Figure 1A) (45). Additionally, glycine affects inflammasome assembly in pro-inflammatory macrophages (46). However, given glycine treatment could induce IKB degradation in resting macrophages (45), we still could not exclude the possibility that glycine causes stress responses in resting macrophages. In addition, in the context of glycine treatment, the decreased phosphorylation of I $\mathrm{KB}$ kinase- $\alpha$ (IKK- $\alpha$ ) and I $\mathrm{K} B$ kinase- $\beta$ (IKK- $\beta$ ) is also observed $(45,46)$ (Figure 1B). Glycine reduces LPS-induced upregulation of nucleotide binding domain like receptor protein 3 (NLRP3) (47). This process can be achieved by up-regulating the expression of NRF2 and its down-stream signaling pathways to eliminate reactive oxygen species (ROS) (47) (Figure 1C).

PI3K (phosphatidylinositol 3-kinase) and Akt (protein kinase B) pathways regulate tremendous signaling pathways, including $\mathrm{NF}-\kappa \mathrm{B}$ and mitogen-activated protein kinase (MAPK) signaling (48) related to macrophage polarization (49). Glycine can upregulate Akt by blocking phosphatase and tensin homolog deleted on chromosome ten (PTEN), then inhibit NF- $\kappa B$ and hypoxia induced factor-1 $\alpha$ (HIF1- $\alpha$ ) in microglia (50) in the context of ischemia-reperfusion injury. Except for macrophages, glycine also inhibits PTEN and activates Akt in other tissues or cells $(51,52)$ (Figure 1D). Unfortunately, there is still no direct evidence showing whether glycine can affect proinflammatory macrophage polarization induced by canonical stimuli (e.g., LPS and/or IFN- $\gamma$ ) through PTEN-Akt pathway. Notably, Akt kinases have distinct effects in macrophage polarization, with Akt1 ablation leading to an M1 phenotype and Akt2 ablation resulting in an M2 phenotype (53). It has not been studied which subunit of Akt is regulated by glycine. Therefore, it is necessary to further explore the connection between glycine and the Akt signaling pathway in guiding macrophages polarization.

\section{GLYCINE ALTERS microRNAs IN MACROPHAGES}

MicroRNAs (miRNAs) play vital roles in a great deal of biological processes (54) and could function as crucial regulators that support macrophage polarization $(54,55)$. It has been reported that some miRNAs which associated with macrophages are related with glycine. For example, glycine alleviates subarachnoid-hemorrhage $(\mathrm{SAH})$ induced neuron inflammation, which is mediated by miRNA-26b/PTEN/Akt signaling pathway in microglia (56) (Figure 2A). Inhibition of miRNA-26b or activation of PTEN expression suppressed the protective function of glycine (56). MiR-301a is abundantly expressed in hypoxic pancreatic cancer cell-derived exosomes $(57,58)$, which can promote M2 macrophage polarization through activating PTEN/PI3K signaling pathway (57). Interestingly, glycine has been reported to enhance the expression of miR-301a in the cortical neurons (59). Thus, miR-301a might be a potential target for glycine to regulate M2 macrophage functions (Figure 2B).

MiR-19a-3p can suppress LPS/IFN- $\boldsymbol{\gamma}$-induced M1 macrophage polarization via inhibiting STAT1 (signal transducer and activator of transcription-1) (60). In addition, glycine regulates miR-19a-3p/AMPK pathway to alleviate ischemic stroke injury (61). Therefore, glycine may promote M1 macrophage polarization by regulating miR-19a-3p (Figure 2C). Besides influencing M1 macrophages polarization, miR-19a-3p is capable of suppressing M2 macrophage polarization by inhibiting STAT3 when overexpressed (62) (Figure 2D).

Notably, miRNAs can regulate GlyTs function. Human GlyT1 possesses several miRNAs targeting sites within the 3'UTR (miR-7, miR-30, miR-96, miR-137, miR-141). Among them, miR-96 and miR-137 negatively regulate GlyT1 under physiological conditions (63) (Figure 2E). It is intriguing to investigate whether microRNAs mediate the regulation of glycinergic system in macrophage polarization.

\section{APPLICATION OF GLYCINE IN MACROPHAGE-RELATED DISEASES}

\section{Obesity and Associated Metabolic Diseases}

The white adipose tissue can produce many adipokines such as leptin, TNF- $\alpha$, and interleukins, due to the accumulation of macrophages (64-66). In adipocytes differentiated 3T3-L1 cells, applying $10 \mathrm{mM}$ glycine in the medium decreases the expression 


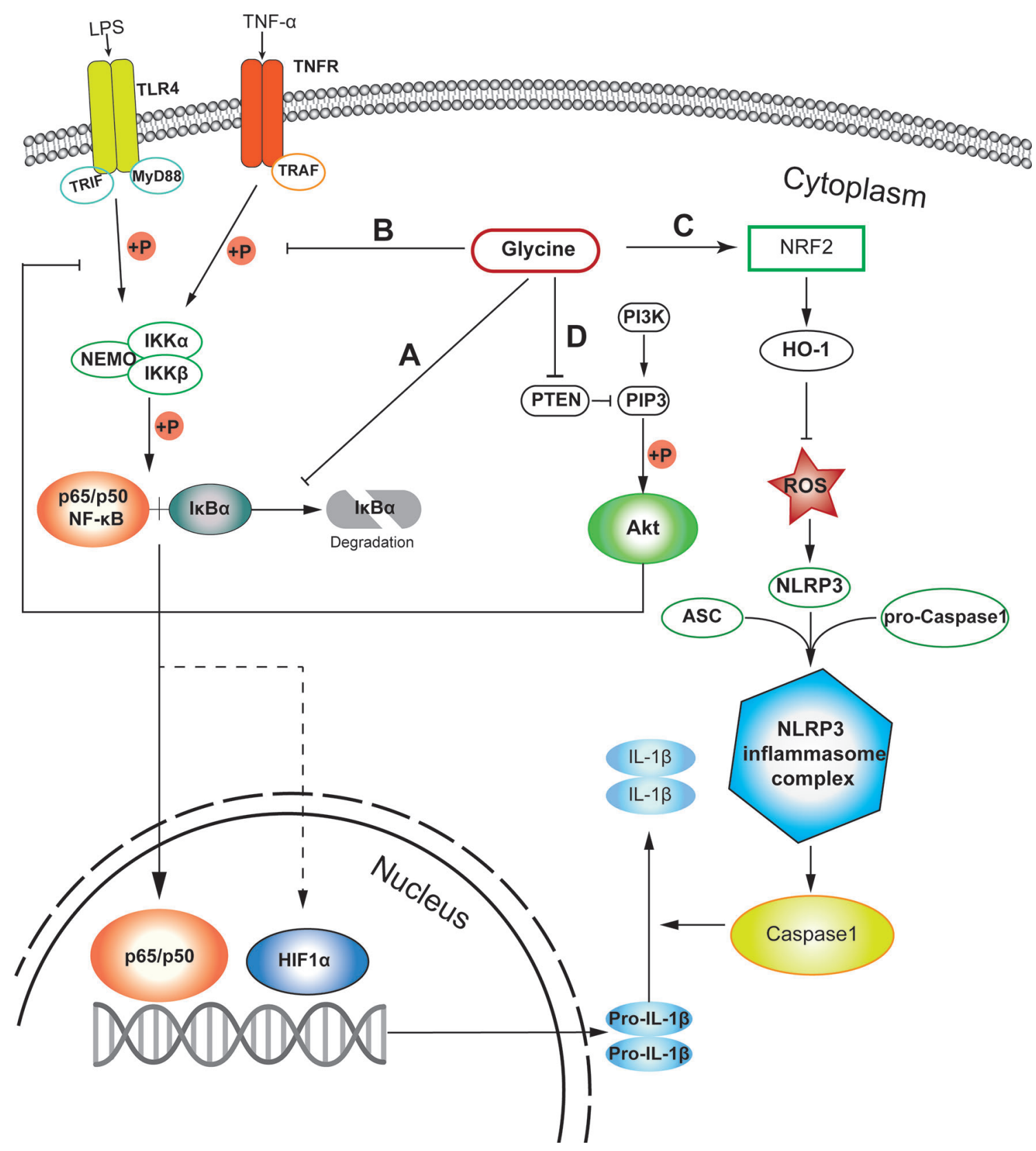

FIGURE 1 | Probable cellular pathways that glycine influences M1 macrophages polarization. (A) Glycine inhibits the degradation of IkB in M1-macrophages. (B) Glycine inhibits M1-macrophages polarization via inhibiting IKK phosphorylation. (C) Glycine up-regulates NRF-2/HO-1 to blunt NLRP3 in inflammasome in M1macrophages. (D) Glycine inhibits NF-кB by blocking PTEN to up-regulate Akt in M1-macrophages. LPS, lipopolysaccharide; TLR4, toll-like receptor 4; MyD88,

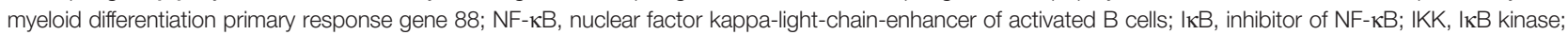
TNF- $\alpha$, tumor necrosis alpha; TNFR, TNF- $\alpha$ receptor; TRAF, TNFR associated factor; PTEN, phosphatase and tensin homolog deleted on chromosome ten; PIP3, phosphatidylinositol $(3,4,5)$-trisphosphate; Akt, protein kinase B.

of IL-6, resistin and TNF- $\alpha$ (67). Similarly, in glutamate-induced obese mice, the application of glycine reprograms fat metabolism and decreases the expression level of TNF- $\alpha$ and IL-6 (68). Serum and liver glycine levels in obese rats are lower than thin rats (69) and dietary supplementation with glycine lowers circulating triglycerides in Zucker fatty rats (70). These phenomena were also found in humans. The plasma glycine level is lower in obese and diabetic patients $(71,72)$ in comparison to healthy donor. In clinical application, dietary supplementation of glycine can improve insulin response and glucose tolerance $(73,74)$. Impaired glycine metabolism may play a causative role in NAFLD, glycine-based treatment 


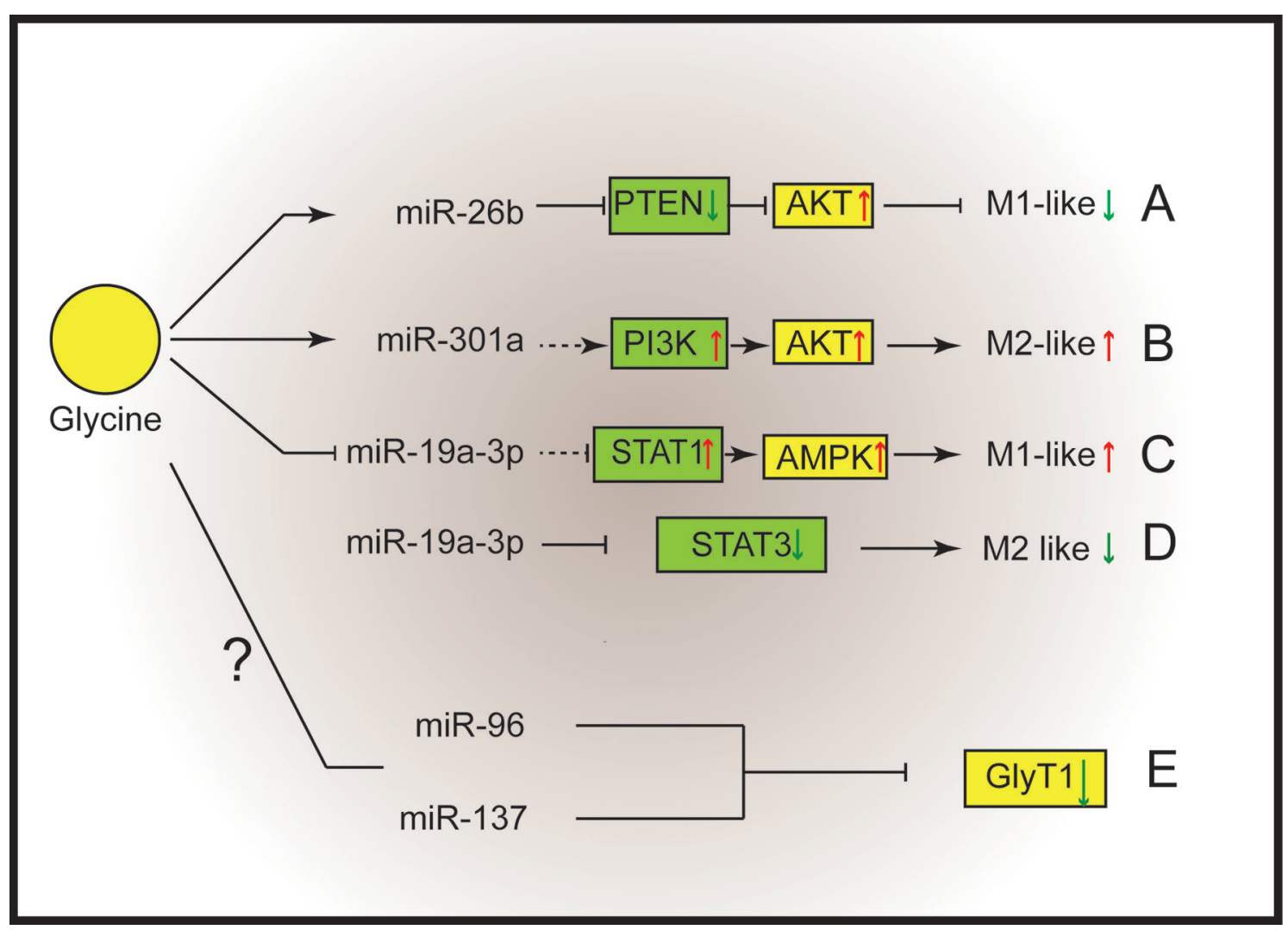

FIGURE 2 | Glycine shapes macrophage polarization through micro-RNAs. (A) Glycine up-regulates miR-26b to blunt M1-microglia polarization by suppressing PTEN and activating Akt. (B) Glycine possibly up-regulates miR-301a to promote M2-macrophages polarization via activating PI3K/Akt. (C, D) Glycine downregulates miR-19a-3p. (C) MiR-19a-3p negatively control STAT1 and AMPK to blunt M1-macrophages polarization. (D) MiR-19a-3p negatively control STAT3 to inhibit M2 macrophage polarization. (E) MiR-96 and miR-137 negatively regulate GlyT1. PTEN, phosphatase and tensin homolog deleted on chromosome ten; Akt, protein kinase B; STAT, signal transducer and activator of transcription; GlyT, glycine transporter.

stimulating hepatic GSH synthesis in experimental NFLD (75). These results show that glycine could be helpful for alleviating inflammatory state in obesity.

Non-alcoholic steatohepatitis (NASH) and non-alcoholic fatty liver disease (NAFLD) are stubborn illnesses because of their prevalence, difficulties in diagnosis, complex pathogenesis, and lack of approved therapies (76). Macrophages are involved in the development of steatosis, inflammation and fibrosis in NASH (77). Furthermore, an increase of M1 macrophages in adipose tissue contribute to NASH due to its secretion of various proinflammatory signals, and these inflammatory factors move to hepatic and trigger local macrophages polarization (78). It has been found that glycine alleviates NASH index in high fat and high sucrose induced NASH in rats (79). Like obesity patients, plasma glycine levels are lower in NAFLD patients (80). Moreover, in a metabolic steatohepatitis mice model, glycine decreases cytokines level and increases M2/M1 macrophages ratio (81). These results indicate that glycine may have potential to treat non-alcoholic hepatic diseases.

Glycine could regulate the intestinal flora and decrease intestine macrophage infiltration in mice under LPS stimulation (82). Interestingly, pro-inflammatory macrophage accumulation was found in obesity humans (83). Besides this, increased pro-inflammatory macrophages were found in the gut of high fat diet (HFD) fed mice (84). Thus, glycine may potential to decrease intestinal pro-inflammatory macrophages infiltration to help alleviating obesity and obesity associated metabolic diseases. Whether glycine can affect intestinal macrophage by affecting intestinal flora needs to be further investigation.

\section{Ischemia-Reperfusion Injury}

Ischemia-reperfusion injury is a serious problem after visceral transplantation $(85,86)$. Glycine significantly increases the survival rate after ischemia-reperfusion and alleviates the inflammatory injury from ischemia-reperfusion. Local perfusion with glycine can alleviate warm ischemia-reperfusion injury in small intestine of rats $(87,88)$ and liver of mice (89-91), as well as renal ischemia reperfusion injury caused by renal hypothermic (92). Interestingly, there exists a solid connection between ischemia-reperfusion injury and macrophages. The activation and migration of macrophages can aggravate inflammation, apoptosis or other stress in apparatus $(93,94)$. 
Fortunately, the researchers found that glycine inhibited the activation of Kupffer cells and their interleukins production during liver ischemia-reperfusion $(89,90,95)$. In short, glycine is helpful for postoperative recovery after ischemia-reperfusion.

\section{Cancer/Tumor}

Tumor associated macrophages (TAMs) are highly prevalent in many solid tumors $(96,97)$. Disrupting the malignant interaction between TAMs and cancer cells may greatly contribute to the survival of cancer patient. However, current targeted therapies of TAMs still fail to give a satisfied effect in tumor control because it is truly difficult to completely clear tumor and simultaneously avoid the high toxicity to patients. Thus, it is urgent to find effective and safe targeted TAM therapies.

Regulating TAMs is one of the targets for cancer treatment. Because of its infinite proliferation ability, cancer cells are highly dependent on glycine and serine uptake for nucleotide synthesis and one-carbon metabolism. Silencing SHMT2 and/or depriving extracellular glycine halts the rapid proliferation of cancer cells, but is not capable of blocking their proliferation completely (97). This phenomenon can rescue by the addition of glycine in the medium (97). Strikingly, glycine is generally consumed by highly proliferative cancer cells, but released by slow-proliferating cells (97). Thus, the demands of glycine may be distinct in different types or different proliferation states of cancer cells. Furthermore, high glycine concentration in tumor microenvironment can be consider as a clinical indicator of poor prognosis of tumor (98). Regulating glycine level in the tumor microenvironment may be an effective treatment for inhibiting the proliferation of cancer cells.

\section{Colitis}

Colitis is an idiopathic intestinal inflammatory disease involving the colon, the clinical manifestations are diarrhea, abdominal pain, and even bloody stools $(99,100)$. Glycine altered colon microbiota and serum amino acids concentration, as well as colon interleukin level in 5\% acetic acid induced colitis in mice (101). Similarly, dietary supplementation of $5 \%$ glycine alleviates colitis induced by 2,4,6-trinitrobenzene sulphonic acid (TNBS) and dextran sulfate sodium (DSS) in rats (102). Besides this, glycine supplementation ameliorates $C$. redentium- induced colitis and enhancing the abundance of Lactobacillus (103). In summary, glycine supplementation may a nutritional strategy to alleviate colitis.
Taken together, these findings suggest that glycine has a certain preventive effect on macrophage-related diseases which are summarized in Table 2. However, the beneficial effects of glycine in other macrophage-associated diseases and the underlying mechanisms still need further investigation.

\section{CONCLUDING REMARKS}

In this review, we introduced glycinergic system in macrophages, and summarized how glycine shapes macrophages polarization. For glycinergic system, GlyRs could be found in macrophages, and the subunits of GlyRs are varied in macrophages with different origins. Though it has been already noted that NAATs exist in macrophages, it is not clear which type of NAATs is expressed in macrophages. Glycine is supposed to affect macrophage through different contributors. Mechanistically, glycine alters macrophage signaling pathways (e.g., NF- $\kappa \mathrm{B}, \mathrm{NRF} 2$, and Akt) and miRNAs. Interestingly, other signaling pathways [e.g., ERK (109)] might also mediate the functions of glycine. Therefore, it is not surprising that glycine could influence the progresses of several macrophageassociated diseases (e.g., colitis and NAFLD).

Indeed, the influences of glycine in macrophage activation are still worth further investigation. Firstly, it is not clear whether glycine can affect methylation reaction in macrophages. In onecarbon metabolism, glycine partly provides the carbon backbones required for the generation of SAM (110), which is the main methyl donor for cellular methylation reaction (39, 111). Recent studies have shown that the methylation of histone (39), DNA (112) or mRNA $(113,114)$ is closely related to macrophage polarization. Therefore, glycine is likely to affect macrophage polarization through methylation modification. Secondly, there are few studies on the effect of glycine on the metabolism of macrophages. Macrophage metabolism is highly related with the function output of macrophages (54). Considering glycine could impact HIF- $1 \alpha$ and mTORC1 that are related to cellular metabolism (e.g., glycolysis), thus studying the effect of glycine on macrophage metabolism is meaningful to reveal the working mechanism of glycine on macrophages function. Finally, studying the effect of glycine on macrophages in the tumor microenvironment may reveal a potential target for cancer therapy. Therefore, it is necessary to find out the

TABLE 2 | Beneficial effects of glycine in other macrophage-associated diseases.

\begin{tabular}{|c|c|c|c|}
\hline Model & Dose & Features & References \\
\hline Arthritis (Rat) & Dietary supplementation with 5\% glycine & Pro-inflammatory cytokines $\downarrow$ & $(104,105)$ \\
\hline Acute pancreatitis (AP) (Rat) & Intravenous injection of 100/300 mmol glycine & $\begin{array}{l}\text { Pathological structure } \uparrow \text {; Pro-inflammatory cytokines } \downarrow \\
\text { MPO activity } \downarrow\end{array}$ & (106) \\
\hline $\begin{array}{l}\text { Oral gingival inflammation } \\
\text { (Cultured gingival epithelial cells) }\end{array}$ & $5 \mathrm{mM}$ glycine supplemented in culture medium & $\begin{array}{l}\text { Pro-inflammatory interleukin level } \downarrow \\
\text { Nf- } \kappa \text { B activation } \downarrow\end{array}$ & $(107)$ \\
\hline Endotoxin (LPS) shock (Rat) & Dietary supplementation with $5 \%$ glycine & $\begin{array}{l}\text { Survival rate } \uparrow \text {, } \\
\text { Serum pro-inflammatory cytokines level } \downarrow\end{array}$ & $(108)$ \\
\hline Colitis (Rat and mice) & Dietary supplementation with $5 \%$ glycine & Macroscopic and histologic scores $\uparrow$ & $(101,102)$ \\
\hline
\end{tabular}

$\uparrow$, increase/up-regulate; $\downarrow$, decrease/down-regulate. 
relationship between glycine, macrophage function and cancer progression.

\section{AUTHOR CONTRIBUTIONS}

ZG wrote the review article. $\mathrm{ZG}, \mathrm{SH}$, and $\mathrm{XW}$ revised the review article. $\mathrm{CH}, \mathrm{JF}$, and $\mathrm{LF}$ helped with designing figures and finding relevant literatures. $\mathrm{MZ}$ and $\mathrm{DX}$ reviewed and revised the

\section{REFERENCES}

1. Dixon LJ, Barnes M, Tang H, Pritchard MT, Nagy LE. Kupffer Cells in the Liver. Compr Physiol (2013) 3:785-97. doi: 10.1002/cphy.c120026

2. Wolf SA, Boddeke HW, Kettenmann H. Microglia in Physiology and Disease. Annu Rev Physiol (2017) 79:619-43. doi: 10.1146/annurevphysiol-022516-034406

3. Murray PJ, Wynn TA. Protective and Pathogenic Functions of Macrophage Subsets. Nat Rev Immunol (2011) 11:723-37. doi: 10.1038/nri3073

4. Epelman S, Lavine KJ, Randolph GJ. Origin and Functions of Tissue Macrophages. Immunity (2014) 41:21-35. doi: 10.1016/j.immuni.2014. 06.013

5. Mills CD, Ley K. M1 and M2 Macrophages: The Chicken and the Egg of Immunity. J Innate Immun (2014) 6:716-26. doi: 10.1159/000364945

6. Shapouri-Moghaddam A, Mohammadian S, Vazini H, Taghadosi M, Esmaeili SA, Mardani F, et al. Macrophage Plasticity, Polarization, and Function in Health and Disease. J Cell Physiol (2018) 233:6425-40. doi: $10.1002 /$ jcp.26429

7. Xia Y, Chen S, Zeng S, Zhao Y, Zhu C, Deng B, et al. Melatonin in Macrophage Biology: Current Understanding and Future Perspectives. J Pineal Res (2019) 66:e12547. doi: 10.1111/jpi.12547

8. Hill AA, Reid Bolus W, Hasty AH. A Decade of Progress in Adipose Tissue Macrophage Biology. Immunol Rev (2014) 262:134-52. doi: 10.1111/ imr.12216

9. Galván-Peña S, O’Neill LA. Metabolic Reprograming in Macrophage Polarization. Front Immunol (2014) 5:420. doi: 10.3389/fimmu.2014.00420

10. Thapa B, Lee K. Metabolic Influence on Macrophage Polarization and Pathogenesis. BMB Rep (2019) 52:360-72. doi: 10.5483/BMBRep.2019. 52.6.140

11. Gogoi M, Datey A, Wilson KT, Chakravortty D. Dual Role of Arginine Metabolism in Establishing Pathogenesis. Curr Opin Microbiol (2016) 29:43-8. doi: 10.1016/j.mib.2015.10.005

12. Gibson NR, Jahoor F, Ware L, Jackson AA. Endogenous Glycine and Tyrosine Production is Maintained in Adults Consuming a MarginalProtein Diet. Am J Clin Nutr (2002) 75:511-8. doi: 10.1093/ajcn/75.3.511

13. Li P, Yin YL, Li D, Kim SW, Wu G. Amino Acids and Immune Function. Br J Nutr (2007) 98:237-52. doi: 10.1017/S000711450769936X

14. Meléndez-Hevia E, De Paz-Lugo P, Cornish-Bowden A, Cárdenas ML. A Weak Link in Metabolism: The Metabolic Capacity for Glycine Biosynthesis Does Not Satisfy the Need for Collagen Synthesis. J Biosci (2009) 34:853-72. doi: 10.1007/s12038-009-0100-9

15. Zhong Z, Wheeler MD, Li X, Froh M, Schemmer P, Yin M, et al. L-Glycine: A Novel Antiinflammatory, Immunomodulatory, and Cytoprotective Agent. Curr Opin Clin Nutr Metab Care (2003) 6:229-40. doi: 10.1097/00075197200303000-00013

16. Wheeler MD, Ikejema K, Enomoto N, Stacklewitz RF, Seabra V, Zhong Z, et al. Glycine: A New Anti-Inflammatory Immunonutrient. Cell Mol Life Sci (1999) 56:843-56. doi: 10.1007/s000180050030

17. Aprison MH, Werman R. The Distribution of Glycine in Cat Spinal Cord and Roots. Life Sci (1965) 4:2075-83. doi: 10.1016/0024-3205(65) 90325-5

18. Wu G, Perlmutter SI. Sensitivity of Spinal Neurons to GABA and Glycine During Voluntary Movement in Behaving Monkeys. J Neurophysiol (2013) 109:193-201. doi: 10.1152/jn.01081.2011 grammar error in the manuscript. All authors contributed to the article and approved the submitted version.

\section{FUNDING}

This study was supported by the Guangdong Basic and Applied Basic Research Foundation (2019B1515210002) and National Natural Science Foundation of China (31922079).

19. Curtis DR, Hösli L, Johnston GA. Inhibition of Spinal Neurons by Glycine Nature (1967) 215:1502-3. doi: 10.1038/2151502a0

20. Betz H, Laube B. Glycine Receptors: Recent Insights Into Their Structural Organization and Functional Diversity. J Neurochem (2006) 97:1600-10. doi: 10.1111/j.1471-4159.2006.03908.x

21. Pfeiffer F, Graham D, Betz H. Purification by Affinity Chromatography of the Glycine Receptor of Rat Spinal Cord. J Biol Chem (1982) 257:9389-93. doi: 10.1016/S0021-9258(18)34082-1

22. Froh M, Thurman RG, Wheeler MD. Molecular Evidence for a GlycineGated Chloride Channel in Macrophages and Leukocytes. Am J Physiol Gastrointest Liver Physiol (2002) 283:G856-63. doi: 10.1152/ ajpgi.00503.2001

23. Carmans S, Hendriks JJ, Thewissen K, Van den Eynden J, Stinissen P, Rigo JM, et al. The Inhibitory Neurotransmitter Glycine Modulates Macrophage Activity by Activation of Neutral Amino Acid Transporters. J Neurosci Res (2010) 88:2420-30. doi: 10.1002/jnr.22395

24. Zhao Y, Zou W, Du J, Zhao Y. The Origins and Homeostasis of Monocytes and Tissue-Resident Macrophages in Physiological Situation. J Cell Physiol (2018) 233:6425-39. doi: 10.1002/jcp.26461

25. Ikejima K, Qu W, Stachlewitz RF, Thurman RG. Kupffer Cells Contain a Glycine-Gated Chloride Channel. Am J Physiol (1997) 272:G1581-6. doi: 10.1152/ajpgi.1997.272.6.G1581

26. Legendre P. The Glycinergic Inhibitory Synapse. Cell Mol Life Sci (2001) 58:760-93. doi: 10.1007/PL00000899

27. Wheeler MD, Thurman RG. Production of Superoxide and TNF-Alpha From Alveolar Macrophages is Blunted by Glycine. Am J Physiol (1999) 277: L952-9. doi: 10.1152/ajplung.1999.277.5.L952

28. Eder C. Ion Channels in Microglia (Brain Macrophages). Am J Physiol (1998) 275:C327-42. doi: 10.1152/ajpcell.1998.275.2.C327

29. Hyde R, Taylor PM, Hundal HS. Amino Acid Transporters: Roles in Amino Acid Sensing and Signalling in Animal Cells. Biochem J (2003) 373:1-18. doi: 10.1042/bj20030405

30. Song W, Li D, Tao L, Luo Q, Chen L. Solute Carrier Transporters: The Metabolic Gatekeepers of Immune Cells. Acta Pharm Sin B (2020) 10:61-78. doi: 10.1016/j.apsb.2019.12.006

31. Schilling T, Eder C. A Novel Physiological Mechanism of Glycine-Induced Immunomodulation: Na+-Coupled Amino Acid Transporter Currents in Cultured Brain Macrophages. J Physiol (2004) 559:35-40. doi: 10.1113/ jphysiol.2004.070763

32. Wang W, Wu Z, Dai Z, Yang Y, Wang J, Wu G. Glycine Metabolism in Animals and Humans: Implications for Nutrition and Health. Amino Acids (2013) 45:463-77. doi: 10.1007/s00726-013-1493-1

33. Amelio I, Cutruzzola F, Antonov A, Agostini M, Melino G. Serine and Glycine Metabolism in Cancer. Trends Biochem Sci (2014) 39:191-8. doi: 10.1016/j.tibs.2014.02.004

34. Arnstein HR, Neuberger A. The Synthesis of Glycine and Serine by the Rat. Biochem J (1953) 55:271-80. doi: 10.1042/bj0550271

35. Locasale JW. Serine, Glycine and One-Carbon Units: Cancer Metabolism in Full Circle. Nat Rev Cancer (2013) 13:572-83. doi: 10.1038/nrc3557

36. Ducker GS, Ghergurovich JM, Mainolfi N, Suri V, Jeong SK, Hsin-Jung Li S, et al. Human SHMT Inhibitors Reveal Defective Glycine Import as a Targetable Metabolic Vulnerability of Diffuse Large B-Cell Lymphoma. Proc Natl Acad Sci USA (2017) 114:11404-9. doi: 10.1073/ pnas. 1706617114 
37. Chen S, Xia Y, He F, Fu J, Xin Z, Deng B, et al. Serine Supports IL-1beta Production in Macrophages Through mTOR Signaling. Front Immunol (2020) 11:1866. doi: 10.3389/fimmu.2020.01866

38. Rodriguez AE, Ducker GS, Billingham LK, Martinez CA, Mainolfi N, Suri V, et al. Serine Metabolism Supports Macrophage IL-1beta Production. Cell Metab (2019) 29:1003-11.e4. doi: 10.1016/j.cmet.2019.01.014

39. Yu W, Wang Z, Zhang K, Chi Z, Xu T, Jiang D, et al. One-Carbon Metabolism Supports S-Adenosylmethionine and Histone Methylation to Drive Inflammatory Macrophages. Mol Cell (2019) 75:1147-60.e5. doi: 10.1016/j.molcel.2019.06.039

40. Jacobs MD, Harrison SC. Structure of an IkappaBalpha/NF-kappaB Complex. Cell (1998) 95:749-58. doi: 10.1016/S0092-8674(00)81698-0

41. Solt LA, May MJ. The IkappaB Kinase Complex: Master Regulator of NF-kappaB Signaling. Immunol Res (2008) 42:3-18. doi: 10.1007/s12026008-8025-1

42. Ivashkiv LB. Ifn $\gamma$ : Signalling, Epigenetics and Roles in Immunity, Metabolism, Disease and Cancer Immunotherapy. Nat Rev Immunol (2018) 18:545-58. doi: 10.1038/s41577-018-0029-z

43. Murray PJ. Macrophage Polarization. Annu Rev Physiol (2017) 79:541-66. doi: 10.1146/annurev-physiol-022516-034339

44. Dorrington MG, Fraser IDC. NF- $\kappa b$ Signaling in Macrophages: Dynamics, Crosstalk, and Signal Integration. Front Immunol (2019) 10:705. doi: 10.3389/fimmu.2019.00705

45. Blancas-Flores G, Alarcón-Aguilar FJ, García-Macedo R, Almanza-Pérez JC, Flores-Sáenz JL, Román-Ramos R, et al. Glycine Suppresses TNF- $\alpha$-Induced Activation of NF- $\kappa \mathrm{b}$ in Differentiated 3T3-L1 Adipocytes. Eur J Pharmacol (2012) 689:270-7. doi: 10.1016/j.ejphar.2012.06.025

46. Contreras-Nuñez E, Blancas-Flores G, Cruz M, Almanza-Perez JC, GomezZamudio JH, Ventura-Gallegosc JL, et al. Participation of the IKK- $\alpha / \beta$ Complex in the Inhibition of the TNF- $\alpha / \mathrm{NF}-\kappa \mathrm{b}$ Pathway by Glycine: Possible Involvement of a Membrane Receptor Specific to Adipocytes. Biomed Pharmacother (2018) 102:120-31. doi: 10.1016/j.biopha.2018.03.048

47. Zhang Y, Ma X, Jiang D, Chen J, Jia H, Wu Z, et al. Glycine Attenuates Lipopolysaccharide-Induced Acute Lung Injury by Regulating NLRP3 Inflammasome and NRF2 Signaling. Nutrients (2020) 12(3):611. doi: 10.3390/nu12030611

48. Liu R, Chen Y, Liu G, Li C, Song Y, Cao Z, et al. PI3K/AKT Pathway as a Key Link Modulates the Multidrug Resistance of Cancers. Cell Death Dis (2020) 11:797. doi: 10.1038/s41419-020-02998-6

49. Haque MA, Jantan I, Harikrishnan H. Zerumbone Suppresses the Activation of Inflammatory Mediators in LPS-Stimulated U937 Macrophages Through MyD88-Dependent NF-кb/MAPK/PI3K-Akt Signaling Pathways. Int Immunopharmacol (2018) 55:312-22. doi: 10.1016/ j.intimp.2018.01.001

50. Liu R, Liao XY, Pan MX, Tang JC, Chen SF, Zhang Y, et al. Glycine Exhibits Neuroprotective Effects in Ischemic Stroke in Rats Through the Inhibition of M1 Microglial Polarization via the NF-кb P65/Hif- $1 \alpha$ Signaling Pathway. J Immunol (2019) 202:1704-14. doi: 10.4049/jimmunol.1801166

51. Zhao D, Chen J, Zhang Y, Liao HB, Zhang ZF, Zhuang Y, et al. Glycine Confers Neuroprotection Through PTEN/AKT Signal Pathway in Experimental Intracerebral Hemorrhage. Biochem Biophys Res Commun (2018) 501:85-91. doi: 10.1016/j.bbrc.2018.04.171

52. Wang M, Yuan F, Bai H, Zhang J, Wu H, Zheng K, et al. SHMT2 Promotes Liver Regeneration Through Glycine-Activated Akt/mTOR Pathway. Transplantation (2019) 103:e188-97. doi: 10.1097/TP.0000000000002747

53. Arranz A, Doxaki C, Vergadi E, Martinez de la Torre Y, Vaporidi K, Lagoudaki ED, et al. Akt1 and Akt2 Protein Kinases Differentially Contribute to Macrophage Polarization. Proc Natl Acad Sci USA (2012) 109:9517-22. doi: 10.1073/pnas.1119038109

54. Xia Y, Li Y, Wu X, Zhang Q, Chen S, Ma X, et al. Ironing Out the Details: How Iron Orchestrates Macrophage Polarization. Front Immunol (2021) 12. doi: 10.3389/fimmu.2021.669566

55. Essandoh K, Li Y, Huo J, Fan GC. MiRNA-Mediated Macrophage Polarization and its Potential Role in the Regulation of Inflammatory Response. Shock (2016) 46:122-31. doi: 10.1097/SHK.0000000000000604

56. Qin X, Akter F, Qin L, Xie Q, Liao X, Liu R, et al. MicroRNA-26b/PTEN Signaling Pathway Mediates Glycine-Induced Neuroprotection in SAH Injury. Neurochem Res (2019) 44:2658-69. doi: 10.1007/s11064-019-02886-2
57. Wang X, Luo G, Zhang K, Cao J, Huang C, Jiang T, et al. Hypoxic TumorDerived Exosomal miR-301a Mediates M2 Macrophage Polarization via PTEN/Pi3k $\gamma$ to Promote Pancreatic Cancer Metastasis. Cancer Res (2018) 78:4586-98. doi: 10.1158/0008-5472.CAN-17-3841

58. Luo G, Xia X, Wang X, Zhang K, Cao J, Jiang T, et al. miR-301a Plays a Pivotal Role in Hypoxia-Induced Gemcitabine Resistance in Pancreatic Cancer. Exp Cell Res (2018) 369:120-8. doi: 10.1016/j.yexcr.2018.05.013

59. Chen J, Zhuang Y, Zhang ZF, Wang S, Jin P, He C, et al. Glycine Confers Neuroprotection Through microRNA-301a/PTEN Signaling. Mol Brain (2016) 9:59. doi: 10.1186/s13041-016-0241-3

60. Zhu X, Guo Q, Zou J, Wang B, Zhang Z, Wei R, et al. MiR-19a-3p Suppresses M1 Macrophage Polarization by Inhibiting STAT1/IRF1 Pathway. Front Pharmacol (2021) 12:614044. doi: 10.3389/fphar.2021.614044

61. Chen ZJ, Zhao XS, Fan TP, Qi HX, Li D. Glycine Improves Ischemic Stroke Through miR-19a-3p/AMPK/GSK-3ß/HO-1 Pathway. Drug Des Devel Ther (2020) 14:2021-31. doi: 10.2147/DDDT.S248104

62. Yang J, Zhang Z, Chen C, Liu Y, Si Q, Chuang TH, et al. MicroRNA-19a-3p Inhibits Breast Cancer Progression and Metastasis by Inducing Macrophage Polarization Through Downregulated Expression of Fra-1 Proto-Oncogene. Oncogene (2014) 33:3014-23. doi: 10.1038/onc.2013.258

63. Jiménez E, Piniella D, Giménez C, Zafra F. Regulation of the Glycine Transporter GLYT1 by microRNAs. Neurochem Res (2021). doi: 10.1007/ s11064-021-03228-x

64. Recasens M, Ricart W, Fernández-Real JM. [Obesity and Inflammation]. Rev Med Univ Navarra (2004) 48:49-54. doi: 10.1155/2007/95974

65. Kanda H, Tateya S, Tamori Y, Kotani K, Hiasa K, Kitazawa R, et al. MCP-1 Contributes to Macrophage Infiltration Into Adipose Tissue, Insulin Resistance, and Hepatic Steatosis in Obesity. J Clin Invest (2006) 116:1494-505. doi: 10.1172/JCI26498

66. Ren W, Xia Y, Chen S, Wu G, Bazer FW, Zhou B, et al. Glutamine Metabolism in Macrophages: A Novel Target for Obesity/Type 2 Diabetes. Adv Nutr (2019) 10:321-30. doi: 10.1093/advances/nmy084

67. Garcia-Macedo R, Sanchez-Muñoz F, Almanza-Perez JC, Duran-Reyes G, Alarcon-Aguilar F, Cruz M. Glycine Increases mRNA Adiponectin and Diminishes Pro-Inflammatory Adipokines Expression in 3T3-L1 Cells. Eur J Pharmacol (2008) 587:317-21. doi: 10.1016/j.ejphar.2008.03.051

68. Almanza-Perez JC, Alarcon-Aguilar FJ, Blancas-Flores G, CamposSepulveda AE, Roman-Ramos R, Garcia-Macedo R, et al. Glycine Regulates Inflammatory Markers Modifying the Energetic Balance Through PPAR and UCP-2. Biomed Pharmacother (2010) 64:534-40. doi: 10.1016/j.biopha.2009.04.047

69. Simmons RM, McKnight SM, Edwards AK, Wu G, Satterfield MC. Obesity Increases Hepatic Glycine Dehydrogenase and Aminomethyltransferase Expression While Dietary Glycine Supplementation Reduces White Adipose Tissue in Zucker Diabetic Fatty Rats. Amino Acids (2020) 52 (10):1413-23. doi: 10.1007/s00726-020-02901-9

70. White PJ, Lapworth AL, McGarrah RW, Kwee LC, Crown SB, Ilkayeva O, et al. Muscle-Liver Trafficking of BCAA-Derived Nitrogen Underlies Obesity-Related Glycine Depletion. Cell Rep (2020) 33:108375. doi: 10.1016/j.celrep.2020.108375

71. Okekunle AP, Li Y, Liu L, Du S, Wu X, Chen Y, et al. Abnormal Circulating Amino Acid Profiles in Multiple Metabolic Disorders. Diabetes Res Clin Pract (2017) 132:45-58. doi: 10.1016/j.diabres.2017.07.023

72. Adeva-Andany M, Souto-Adeva G, Ameneiros-Rodríguez E, FernándezFernández C, Donapetry-García C, Domínguez-Montero A. Insulin Resistance and Glycine Metabolism in Humans. Amino Acids (2018) 50:11-27. doi: 10.1007/s00726-017-2508-0

73. Gannon MC, Nuttall JA, Nuttall FQ. The Metabolic Response to Ingested Glycine. Am J Clin Nutr (2002) 76:1302-7. doi: 10.1093/ajcn/76.6.1302

74. Alves A, Bassot A, Bulteau AL, Pirola L, Morio B. Glycine Metabolism and Its Alterations in Obesity and Metabolic Diseases. Nutrients (2019) 11 (6):1356. doi: 10.3390/nu11061356

75. Rom O, Liu Y, Liu Z, Zhao Y, Wu J, Ghrayeb A, et al. Glycine-Based Treatment Ameliorates NAFLD by Modulating Fatty Acid Oxidation, Glutathione Synthesis, and the Gut Microbiome. Sci Transl Med (2020) 12 (572):eaaz2841. doi: 10.1126/scitranslmed.aaz2841

76. Neuschwander-Tetri BA. Non-Alcoholic Fatty Liver Disease. BMC Med (2017) 15:45. doi: 10.1186/s12916-017-0806-8 
77. Kazankov K, Jørgensen SMD, Thomsen KL, Møller HJ, Vilstrup H, George J, et al. The Role of Macrophages in Nonalcoholic Fatty Liver Disease and Nonalcoholic Steatohepatitis. Nat Rev Gastroenterol Hepatol (2019) 16:14559. doi: 10.1038/s41575-018-0082-x

78. Alisi A, Carpino G, Oliveira FL, Panera N, Nobili V, Gaudio E. The Role of Tissue Macrophage-Mediated Inflammation on NAFLD Pathogenesis and Its Clinical Implications. Mediators Inflamm (2017) 2017:8162421. doi: $10.1155 / 2017 / 8162421$

79. Zhou X, Han D, Xu R, Wu H, Qu C, Wang F, et al. Glycine Protects Against High Sucrose and High Fat-Induced non-Alcoholic Steatohepatitis in Rats. Oncotarget (2016) 7:80223-37. doi: 10.18632/oncotarget.12831

80. Takashima S, Ikejima K, Arai K, Yokokawa J, Kon K, Yamashina S, et al. Glycine Prevents Metabolic Steatohepatitis in Diabetic KK-Ay Mice Through Modulation of Hepatic Innate Immunity. Am J Physiol Gastrointest Liver Physiol (2016) 311:G1105-13. doi: 10.1152/ ajpgi.00465.2015

81. Zhang Y, Mu T, Jia H, Yang Y, Wu Z. Protective Effects of Glycine Against Lipopolysaccharide-Induced Intestinal Apoptosis and Inflammation. Amino Acids (2021). doi: 10.1007/s00726-021-03011-w

82. Rohm TV, Fuchs R, Müller RL, Keller L, Baumann Z, Bosch AJT, et al. Obesity in Humans Is Characterized by Gut Inflammation as Shown by ProInflammatory Intestinal Macrophage Accumulation. Front Immunol (2021) 12.

83. Rohm TV, Alasfoor S, Bosch AJ, Cavelti-Weder C. Targeting Intestinal Macrophages as a Potential Therapeutic Option in Obesity. Diabetes (2018) 67:283-OR. doi: 10.2337/db18-283-OR

84. Kahn J, Schemmer P. Control of Ischemia-Reperfusion Injury in Liver Transplantation: Potentials for Increasing the Donor Pool. Visc Med (2018) 34:444-8. doi: 10.1159/000493889

85. Mehrabi A, Mood Zh A, Sadeghi M, Schmied BM, Müller SA, Welsch T, et al. Thymoglobulin and Ischemia Reperfusion Injury in Kidney and Liver Transplantation. Nephrol Dial Transplant (2007) 22(Suppl 8):viii54-60. doi: $10.1093 / \mathrm{ndt} / \mathrm{gfm} 651$

86. Lee MA, McCauley RD, Kong SE, Hall JC. Influence of Glycine on Intestinal Ischemia-Reperfusion Injury. JPEN J Parenter Enteral Nutr (2002) 26:130-5. doi: $10.1177 / 0148607102026002130$

87. Lee MA, McCauley RD, Kong SE, Hall JC. Pretreatment With Glycine Reduces the Severity of Warm Intestinal Ischemic-Reperfusion Injury in the Rat. Ann Plast Surg (2001) 46:320-6. doi: 10.1097/00000637-20010300000020

88. Habib MM, Hodgson HJ, Davidson BR. The Role of Glycine in Hepatic Ischemia-Reperfusion Injury. Curr Pharm Des (2006) 12:2953-67. doi: $10.2174 / 138161206777947605$

89. Yamanouchi K, Eguchi S, Kamohara Y, Yanaga K, Okudaira S, Tajima Y, et al. Glycine Reduces Hepatic Warm Ischaemia-Reperfusion Injury by Suppressing Inflammatory Reactions in Rats. Liver Int (2007) 27:1249-54. doi: 10.1111/j.1478-3231.2007.01564.x

90. Ito K, Ozasa H, Noda Y, Koike Y, Arii S, Horikawa S. Effect of nonEssential Amino Acid Glycine Administration on the Liver Regeneration of Partially Hepatectomized Rats With Hepatic Ischemia/ Reperfusion Injury. Clin Nutr (2008) 27:773-80. doi: 10.1016/ j.clnu.2008.06.012

91. Mangino MJ, Murphy MK, Grabau GG, Anderson CB. Protective Effects of Glycine During Hypothermic Renal Ischemia-Reperfusion Injury. Am J Physiol (1991) 261:F841-8. doi: 10.1152/ajprenal.1991.261.5.F841

92. Liu A, Fang H, Dirsch O, Jin H, Dahmen U. Early Release of Macrophage Migration Inhibitory Factor After Liver Ischemia and Reperfusion Injury in Rats. Cytokine (2012) 57:150-7. doi: 10.1016/ j.cyto.2011.11.009

93. Gazoni LM, Tribble CG, Zhao MQ, Unger EB, Farrar RA, Ellman PI, et al. Pulmonary Macrophage Inhibition and Inhaled Nitric Oxide Attenuate Lung Ischemia-Reperfusion Injury. Ann Thorac Surg (2007) 84:247-53. doi: 10.1016/j.athoracsur.2007.02.036

94. Al-Saeedi M, Liang R, Schultze DP, Nickkholgh A, Herr I, Zorn M, et al. Glycine Protects Partial Liver Grafts From Kupffer Cell-Dependent Ischemia-Reperfusion Injury Without Negative Effect on Regeneration. Amino Acids (2019) 51:903-11. doi: 10.1007/s00726-019-02722-5
95. Räihä MR, Puolakkainen PA. Tumor-Associated Macrophages (TAMs) as Biomarkers for Gastric Cancer: A Review. Chronic Dis Transl Med (2018) 4:156-63. doi: 10.1016/j.cdtm.2018.07.001

96. Gambardella V, Castillo J, Tarazona N, Gimeno-Valiente F, MartínezCiarpaglini C, Cabeza-Segura M, et al. The Role of Tumor-Associated Macrophages in Gastric Cancer Development and Their Potential as a Therapeutic Target. Cancer Treat Rev (2020) 86:102015. doi: 10.1016/ j.ctrv.2020.102015

97. Jain M, Nilsson R, Sharma S, Madhusudhan N, Kitami T, Souza AL, et al. Metabolite Profiling Identifies a Key Role for Glycine in Rapid Cancer Cell Proliferation. Science (2012) 336:1040-4. doi: 10.1126/science.1218595

98. Redalen KR, Sitter B, Bathen TF, Grøholt KK, Hole KH, Dueland S, et al. High Tumor Glycine Concentration is an Adverse Prognostic Factor in Locally Advanced Rectal Cancer. Radiother Oncol (2016) 118:393-8. doi: 10.1016/j.radonc.2015.11.031

99. Lazenby AJ, Giardiello FM, Bayless TM, Yardley JH. Inflammatory Bowel Disease. N Engl J Med (1992) 326:574. doi: 10.1056/ NEJM199202203260815

100. Suzuki T. Regulation of Intestinal Epithelial Permeability by Tight Junctions. Cell Mol Life Sci (2013) 70:631-59. doi: 10.1007/s00018-012-1070-x

101. Wu X, Zheng Y, Ma J, Yin J, Chen S. The Effects of Dietary Glycine on the Acetic Acid-Induced Mouse Model of Colitis. Mediators Inflammation (2020) 2020:5867627. doi: 10.1155/2020/5867627

102. Tsune I, Ikejima K, Hirose M, Yoshikawa M, Enomoto N, Takei Y, et al. Dietary Glycine Prevents Chemical-Induced Experimental Colitis in the Rat. Gastroenterology (2003) 125:775-85. doi: 10.1016/S0016-5085(03) 01067-9

103. Zhang Y, Jiang D, Jin Y, Jia H, Yang Y, Kim IH, et al. Glycine Attenuates Citrobacter Rodentium-Induced Colitis by Regulating ATF6-Mediated Endoplasmic Reticulum Stress in Mice. Mol Nutr Food Res (2021) 65: e2001065. doi: $10.1002 / \mathrm{mnfr} .202001065$

104. Li X, Bradford BU, Wheeler MD, Stimpson SA, Pink HM, Brodie TA, et al. Dietary Glycine Prevents Peptidoglycan Polysaccharide-Induced Reactive Arthritis in the Rat: Role for Glycine-Gated Chloride Channel. Infect Immun (2001) 69:5883-91. doi: 10.1128/IAI.69.9.5883-5891.2001

105. Vieira CP, De Oliveira LP, Da Ré Guerra F, Dos Santos De Almeida M, Marcondes MC, Pimentel ER. Glycine Improves Biochemical and Biomechanical Properties Following Inflammation of the Achilles Tendon. Anat Rec (Hoboken) (2015) 298:538-45. doi: 10.1002/ar.23041

106. Ceyhan GO, Timm AK, Bergmann F, Günther A, Aghdassi AA, Demir IE, et al. Prophylactic Glycine Administration Attenuates Pancreatic Damage and Inflammation in Experimental Acute Pancreatitis. Pancreatology (2011) 11:57-67. doi: 10.1159/000325972

107. Schaumann T, Kraus D, Winter J, Wolf M, Deschner J, Jäger A. Potential Immune Modularly Role of Glycine in Oral Gingival Inflammation. Clin Dev Immunol (2013) 2013:808367. doi: 10.1155/2013/808367

108. Ikejima K, Iimuro Y, Forman DT, Thurman RG. A Diet Containing Glycine Improves Survival in Endotoxin Shock in the Rat. Am J Physiol (1996) 271: G97-103. doi: 10.1152/ajpgi.1996.271.1.G97

109. Ren W, Liu G, Chen S, Yin J, Wang J, Tan B, et al. Melatonin Signaling in T Cells: Functions and Applications. J Pineal Res (2017) 62:e12394. doi: $10.1111 /$ jpi.12394

110. Ly CH, Lynch GS, Ryall JG. A Metabolic Roadmap for Somatic Stem Cell Fate. Cell Metab (2020) 31:1052-67. doi: 10.1016/j.cmet.2020.04.022

111. Newman AC, Maddocks ODK. Serine and Functional Metabolites in Cancer. Trends Cell Biol (2017) 27:645-57. doi: 10.1016/ j.tcb.2017.05.001

112. Wang X, Cao Q, Yu L, Shi H, Xue B, Shi H. Epigenetic Regulation of Macrophage Polarization and Inflammation by DNA Methylation in Obesity. JCI Insight (2016) 1:e87748. doi: 10.1172/jci.insight.87748

113. Liu Y, Liu Z, Tang H, Shen Y, Gong Z, Xie N, et al. The N6Methyladenosine (M6a)-Forming Enzyme METTL3 Facilitates M1 Macrophage Polarization Through the Methylation of STAT1 mRNA Am J Physiol Cell Physiol (2019) 317:C762-75. doi: 10.1152/ ajpcell.00212.2019

114. Yin H, Zhang X, Yang P, Zhang X, Peng Y, Li D, et al. RNA M6a Methylation Orchestrates Cancer Growth and Metastasis via Macrophage 
Reprogramming. Nat Commun (2021) 12:1394. doi: 10.1038/s41467-02121514-8

Conflict of Interest: The authors declare that the research was conducted in the absence of any commercial or financial relationships that could be construed as a potential conflict of interest.

Publisher's Note: All claims expressed in this article are solely those of the authors and do not necessarily represent those of their affiliated organizations, or those of the publisher, the editors and the reviewers. Any product that may be evaluated in this article, or claim that may be made by its manufacturer, is not guaranteed or endorsed by the publisher.

Copyright $(2021$ Gan, Zhang, Xie, Wu, Hong, Fu, Fan, Wang and Han. This is an open-access article distributed under the terms of the Creative Commons Attribution License (CC BY). The use, distribution or reproduction in other forums is permitted, provided the original author(s) and the copyright owner(s) are credited and that the original publication in this journal is cited, in accordance with accepted academic practice. No use, distribution or reproduction is permitted which does not comply with these terms. 